\title{
El vídeo casero: Parámetros formales y de contenido
}

\author{
Laura PICAZO SÁNCHEZ \\ laura.picazo.sanchez@gmail.com \\ Doctoranda en medios audiovisuales-Sección Departamental de Biblioteconomía y \\ Documentación y Departamento Comunicación Audiovisual y Publicidad II \\ Universidad Complutense de Madrid
}

Recibido: $15 / 04 / 2013$

Aceptado: 05/05/2013

\begin{abstract}
RESUMEN
Los procesos de comunicación actuales, cuyo abanico de posibilidades se ha disparado gracias a Internet 2.0, unidos a la accesibilidad a los dispositivos de captación de imágenes en vídeo hacen que la emergencia de contenidos audiovisuales creados por los no expertos haya obtenido un éxito progresivo y una importancia como género. La bidireccionalidad en la red ofrece la posibilidad de publicar las propias grabaciones y consumir otras de forma sencilla y gratuita, favoreciendo un libre flujo de videocreaciones de todo tipo. Sin embargo, estas piezas amateur conviven con otras profesionales y, debido a la mejora en la calidad de los dispositivos y al fácil empleo de las herramientas de edición, se confunden y equivocan al estudioso, al creador y al espectador. Es necesario conocer qué diferencia a los vídeos caseros de todos los demás y poder así identificarlos y considerarlos como un género independiente, fruto de una evolución tecnológica pero también de una asimilación del audiovisual profesional.
\end{abstract}

Palabras clave: video casero, definición, YouTube, amateur, videoaficionado.

\section{Home made video: formal and content criteria}

\begin{abstract}
Current communication process, whom possibilities are exploded because of 2.0 , plus the accesibility to diseases for catching images (nowadays in every person's pocket), make that audiovisual content emerge, created by amateurs, has gotten a progressive success and a care as genre. Bidirectionality on line offers publishing own records and consuming others by simple and free ways, working on a free videocreations fluxus way. However, that contents live with another professionals creations that, because of the quality of the cameras and new easy edition tools, misunderstand and confuse the researchers, producers and users. It's necessary to know which differences exists between amateurs video records and all others to identify them and to consider them as a genre by themselves, products of a technological evolution but also products of audiovisual learning by new creators.
\end{abstract}

Keywords: home made video, definition, YouTube, amateur, video fan. 


\section{INTRODUCCIÓN}

La investigación plasmada en este artículo fue motivada por la fascinación que despierta en el panorama de los géneros audiovisuales un producto conocido cuyo resurgimiento se traduce en un nuevo concepto que se está propagando por todos los medios de comunicación audiovisual y que hace necesaria, a nuestro juicio, una observación detenida. De rabiosa actualidad y creciente interés, las motivaciones del consumidor y creador de vídeo casero, la autoimagen del usuario en Internet y su relación con la clave del fenómeno viral requieren de una identificación científica de los contenidos que los componen. Por eso, este estudio se ha orientado a tratar de identificar el vídeo casero como género distinguible de otros.

Conocer la naturaleza específica de este género se constituyó en el objetivo primordial sobre el supuesto de que no existe ningún estudio previo sobre el vídeo casero y su definición. Observamos que la mayor parte de las alusiones se hacían a lo que popularmente se entiende como vídeo casero: una pieza de baja calidad grabada por un inexperto en el entorno del hogar. No obstante, si aspirábamos a poder identificar estas piezas con cierto rigor y claridad, no podíamos ceñirnos a las aproximaciones no contrastadas que sobre este género se habían realizado. Además, el fenómeno YouTube y la constante referencia que medios y usuarios hacen de la plataforma como archivo audiovisual por excelencia acentúan la necesidad de descomponer el género casero y hallar sus claves de contenido, pues la facilidad y gratuidad de YouTube han explotado las posibilidades de estas piezas y su alcance.

\subsection{Un género indeterminado}

Pese a que incluso los manuales de tecnología videográfica contemplan un tipo de grabaciones que denominan "casero", no existe ninguna tipología contrastada, convenientemente estudiada y válida como para basar una definición siquiera ajustada a la realidad de una categoría de vídeo que podamos denominar casero.

Se han encontrado, sin embargo, múltiples referencias a este género y a su forma y contenido, sobre todo a raíz de su éxito en Internet:

"Se denomina vídeo doméstico o casero a las películas realizadas por aficionados. Desde que se inició esta actividad, las películas caseras se grababan en cintas fotográficas, pero con el tiempo la disponibilidad de videocámaras y dispositivos de almacenamiento de datos ha hecho que este pasatiempo sea más sencillo y accesible a las personas comunes. Los límites entre la creación de vídeos para la gente común y para profesionales se han desdibujado cada vez más, a la vez que el equipamiento del productor/consumidor se hace más accesible al consumidor individual, ofreciendo características y posibilidades que antes solo estaban disponibles para los equipos operados por profesionales. En los últimos años los vídeos grabados por aficionados se han emitido a audiencias masivas a través de programas como America's Funniest Home Videos y el sitio web para compartir vídeos YouTube. Este último, sumado a la popularidad de Internet y una mayor disponibilidad de conexiones de mayor 
velocidad, han permitido proveer y desarrollar nuevas formas de compartir esta clase de vídeos, tales como blogs y podcasting". Wikipedia (12/9/2010). Entendemos que, a partir de descripciones como ésta, puede desarrollarse un estudio que contemple las múltiples formas de vídeo casero y sirva para diferenciarlo de otro tipo de obras desde el punto de vista técnico y de contenido, acercándonos en lo posible a una definición del género más concreta y clara que las existentes hasta hoy.

\subsection{Superproducción audiovisual}

Cuando nos referimos a la era audiovisual tratamos de poner nombre a un proceso de invasión consentida que la imagen ha efectuado en la vida del individuo. Alcanzar el máximo exponente de sus funciones y capacidades hasta ahora conocido sólo es posible con el desarrollo de los medios de comunicación y la evolución del 2.0, una extensión de Internet que catapulta la interacción entre el usuario y la red, alterando los roles de consumidor y productor y generando un libre flujo de contenidos entre usuarios que, hasta el 2.0, estaban condenados a una posición pasiva con respecto al medio.

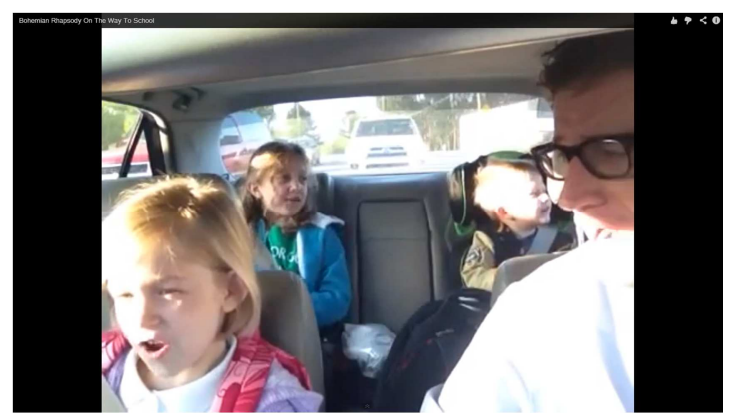

Imagen 1: Bohemian Rhapsody on the way to school

Mencionar este cambio de panorama nos ayuda a introducir la sobreexposición del individuo a la imagen. Hoy, la superproducción de imágenes fijas y en movimiento afecta al ritmo y a la atención con que asimilamos sus mensajes, pero no concebimos la comunicación sin ellas. Y es de ellas de donde extraemos nuestros propios modelos para crear.

\section{MÉTODO DE TRABAJO}

"Si no se hace este estudio, el investigador actúa de acuerdo con su gusto personal, y la división objetiva de los temas resulta simplemente imposible" (BARTHES, 1970) "He aquí, pues, la cuarta tesis fundamental de nuestro trabajo: Todos los cuentos maravillosos pertenecen al mismo tipo en lo que concierne a su estructura" (PROPP, 1928) 
De La morfología del cuento (Propp, 1928) hemos aprendido que es necesario delimitar una clase de productos culturales analizando su composición para conocer qué tiene de particular o distintivo respecto de otros productos: en su caso el "cuento maravilloso" y en el nuestro el "video casero" es el objeto material de estudio. Nuestro objetivo general es proponer una definición del vídeo casero en torno a sus características técnicas y a sus elementos de contenido más habituales. "Es incontestable que se pueden estudiar los fenómenos y los objetos que nos rodean desde el punto de vista de su composición y su estructura, desde el punto de vista de los procesos y las transformaciones a que están sometidos. Hay otra evidencia más, que no precisa demostración alguna: no se puede hablar del origen de un fenómeno, sea el que sea, antes de describir este fenómeno" (Propp, 1928:11). Entendemos que el conocimiento de su composición (al menos, hasta cierto nivel de detalle) es importante en sí mismo.

Para el estudio de la estructura del "video casero", hemos tomado como modelo el análisis pionero de Vladimir Propp. Como él hizo con el cuento maravilloso, nosotros nos hemos propuesto hallar las constantes que cabe encontrar en la estructura de los vídeos caseros, independientemente de su procedencia, de la relación entre sus autores y de otros aspectos como la fecha de grabación, la publicación o no publicación del contenido, la difusión, el número de visitas, la duración o el relato que en ellos se desarrolla ${ }^{1}$.

Asimismo, hemos tomado como ejemplo la aplicación que otros estudiosos de los relatos ejercieron de los trabajos de Propp, (como Jesús González Requena, para su análisis de los géneros cinematográficos clásico, manierista y postclásico ${ }^{2}$, José Luis Piñuel Raigada, para el estudio de las novenas populares y Javier Sánchez Carrión, que se basó en esta metodología para identificar las claves del telefilm policíaco).

\footnotetext{
1 "Se suele llamar análisis de contenido al conjunto de procedimientos interpretativos de productos comunicativos (mensajes, textos o discursos) que proceden de procesos singulares de comunicación previamente registrados, y que, basados en técnicas de medida, a veces cuantitativas (estadísticas basadas en el recuento de unidades), a veces cualitativas (lógicas basadas en la combinación de categorías) tienen por objeto elaborar y procesar datos relevantes sobre las condiciones mismas en que se han producido aquellos textos, o sobre las condiciones que puedan darse para su empleo posterior. El análisis de contenido, de hecho, se convirtió a finales del siglo XX en una de las técnicas de uso más frecuente en muchas ciencias sociales, adquiriendo una relevancia desconocida en el pasado a medida que se introdujeron procedimientos informáticos en el tratamiento de los datos." Piñuel (2002:2).

2 "Propp constata cómo, más allá de las variaciones de los personajes, cierta estructura constante de acciones caracteriza al conjunto de los cuentos que constituyen su corpus: “(...) Los elementos constantes, permanentes, del cuento son las funciones de los personajes, sean cuales fueren estos personajes y sea cual sea la manera en que cumplan esas funciones. Las funciones son las partes constitutivas fundamentales del cuento." A través de un examen inductivo sistemático de su corpus, Propp aísla las unidades estructurales del cuento maravilloso, demostrando que éste constituye un sistema narrativo altamente estructurado. En él "(...) las funciones se repiten de una manera asombrosa." (González Requena, 2006:507).
} 


\subsection{Particularidades del estudio del video casero}

En todos los casos señalados, se pretende reconocer las constantes de todas las piezas que forman parte de un conjunto, con el fin de identificar la uniformidad de ese conjunto y, consecuentemente, poder definirlo desde el punto de vista de su estructura. Sin embargo, el objeto de estudio de este trabajo es sustancialmente distinto, y conlleva, por eso mismo, una perspectiva matizada del análisis de contenido. Si bien los modelos metodológicos en el caso de Propp ${ }^{3}$ y los otros autores citados plantean hallar a través del análisis de contenido unas funciones específicas de los personajes, cronológicamente registradas en los relatos y comunes en todos los productos de cada tipología, en el caso del vídeo casero la designación del relato se lleva a cabo por unas cualidades técnicas y relacionadas con la ejecución del vídeo, y no por aquellas que atañen exclusivamente a la diégesis y a los personajes, y que no pueden, por tanto, hallarse de forma cronológica en el relato videográfico casero.

\section{LA INVESTIGACIÓN}

La dimensión técnica constituye un elemento decisivo, pues son estas características las que han guiado las definiciones del vídeo casero hasta ahora conocidas. Nosotros hemos atendido también a la dimensión del contenido pues de esta observación se ha podido extraer una tendencia en el género. La investigación está basada en el sometimiento de un corpus de vídeos (todavía aparentemente) caseros a un protocolo de análisis que presentaremos en este mismo capítulo.

\subsection{Procedimiento}

El primer paso consistió en la elaboración de un modelo de Ficha de Registro (Protocolo de Análisis) al que aplicar la muestra y con el que obtener tantas fichas de registro como unidades componen la muestra.

Para confeccionar esta ficha fue preciso averiguar antes qué preguntas necesitábamos hacer a cada pieza con el fin de alcanzar nuestros objetivos. Basándonos en los modelos analíticos institucionalizados de otros géneros audiovisuales (televisivos y cinematográficos) examinamos, desde las perspectivas formal y narrativa, diez piezas aparentemente caseras que superaban el millón de visitas en YouTube. Así, los análisis contemplan:

- Duración

- Resumen del contenido

- Personajes

\footnotetext{
${ }^{3}$ En González Requena (2006:528).
} 
- Acción principal ${ }^{4}$

- Final

- Diálogos

- Temática

- Características de la imagen

- Características del sonido

- Características del montaje/ edición

- Música

- Idioma

- Autor/ Referencia

- Fecha de publicación

- Respuestas al vídeo

- Número de visitas

\section{Ficha de registro}

Tras el análisis de los diez vídeos del ensayo, sintetizamos en conceptos los aspectos a los que se había atendido. Dichos núcleos temáticos fueron clave para desarrollar la Ficha de Registro:

-Contenido - Cuál es la acción principal desarrollada en el mismo, personajes, diálogo, resolución de la acción.

-Forma - Cuáles son las características de la grabación, qué calidad tienen la imagen y el sonido, cómo se maneja la cámara; cómo son los cortes y el montaje (si el vídeo los registra).

-Difusión - El vídeo ha sido publicado en YouTube pero, ¿se sabe quién lo ha difundido? ¿Está relacionado con la grabación? ¿Qué comentarios ha hecho el difusor?

-Repercusión - Número de visitas del vídeo, vídeos doblados ${ }^{5}$.

${ }^{4}$ Al plantearnos observar la acción, hemos de poder definirla y concluimos que, teniendo en cuenta la escasa duración de los vídeos caseros a analizar -piezas de un máximo de ocho minutos tomaremos la acción más destacada, más representativa o llamativa.

${ }^{5}$ Por vídeos doblados entendemos vídeos que han sido copiados y publicados repetidas veces,

llegando a tal punto que resulta muy difícil hallar el vídeo original, ya que se permite alterar el título $\mathrm{y}$, al hacerse la búsqueda por tags (etiquetas atribuibles a la pieza que se aplican al buscador para localizarla; por ejemplo: caída, risa, niño...), cada vídeo, pese a ser doblado, puede tener sus propios tags, de manera que al comparar las fechas de subida de todos ellos, no podemos saber si existen más vídeos con el mismo contenido, convirtiéndose en una búsqueda aleatoria. 
La Ficha de Registro es un documento intermedio entre el producto que se analiza y la base de datos donde se transcribirán finalmente las observaciones una vez codificadas.

\section{Libro de códigos}

Finalizada la elaboración de la Ficha de Registro, era necesario complementarla con un manual de instrucciones de uso. El libro de códigos desempeña una función explicativa que sirve al analista para aplicar correctamente la Ficha de Registro a cada una de las unidades del corpus.

En él se detalla cómo han de visualizarse los vídeos de la muestra y cómo completar los datos de referencia (nombre del vídeo, fecha del análisis) de cada unidad de registro.

\subsection{La muestra}

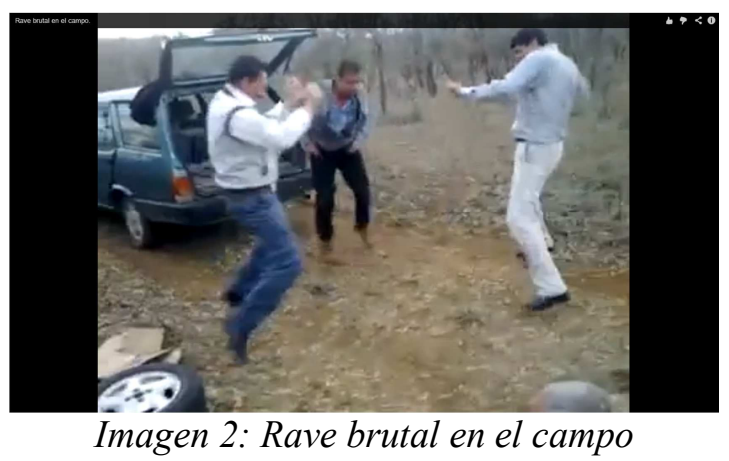

El corpus de este estudio está compuesto por 30 vídeos, de los cuales:

- Veinte son vídeos aparentemente caseros, esto es, cumplen los requisitos técnicos que, según los parámetros teóricos existentes, caracterizan al vídeo casero: vídeos de baja calidad técnica, tanto visual como sonora, cuya captación pareciera realizada con un dispositivo doméstico o con webcam.

De ellos, dieciocho han sido visitados más de 10.000 veces.

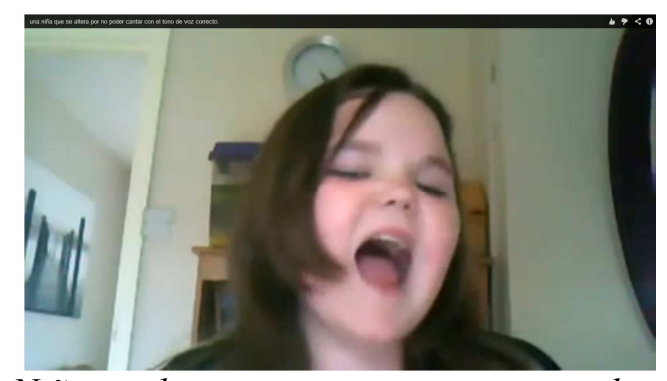

Imagen 3: Niña se altera por no cantar con tono de voz correcto 
- Diez son vídeos profesionales, pero, de estos diez, cuatro cumplen requisitos técnicos caseros. La elección de esta parte de la muestra se hizo intencionadamente, tratando de incluir en ella ejemplos de los vídeos profesionales más parecidos a los caseros, en un intento por reflejar, en las conclusiones finales, parámetros objetivos realmente efectivos en la diferenciación del género.

Los resultados del análisis de estos diez vídeos profesionales no se procesaron del mismo modo que el resto de vídeos de la muestra (los 20 aparentemente caseros). De las conclusiones extraídas de este sector profesional separamos los elementos comunes con el vídeo casero, descartando dichos parámetros como elementos identificativos del género amateur.

\subsection{Experiencia}

Tras la aplicación del protocolo a la muestra, se obtuvieron los resultados de la experiencia, que determinó qué elementos son más frecuentes ${ }^{6}$ y cuáles menos. De esta diferencia extrajimos una serie de posibles características definitivas que sometimos a una segunda observación: reflexionar, una a una y con ayuda de la base de datos obtenida qué información aportan y qué utilidad tienen para diferenciar a los vídeos caseros del resto de obras audiovisuales.

Para hacer más transparente esta explicación, vamos a traducir en preguntas los criterios que permiten calificar a una característica como útil para diferenciar al vídeo casero de cualquier otra pieza profesional:

-¿Puede darse este elemento en una obra profesional de baja calidad de imagen y/o sonido?

-¿Puede darse este elemento en una obra profesional que recoja un instante cualquiera de la vida cotidiana?

-¿Puede darse este elemento en una obra profesional que intente emular un vídeo de fabricación casera?

Pese a que, de los 69 que componían la Ficha de Registro, contábamos con 39 elementos comunes a los "vídeos caseros" de la muestra, sólo once eran representativos del género casero y sólo ellos resultan en la práctica efectivos para diferenciar las obras amateur de aquellas realizadas por profesionales.

\footnotetext{
${ }^{6}$ Denominaremos frecuente a aquel parámetro que supere una representatividad de 4 de cada 10 en la muestra.
} 


\section{RESULTADOS}

\subsection{Elementos del video casero}

Decidimos, para alcanzar nuestro objetivo, convertir los resultados en las características más comunes del vídeo casero, que denominaremos a partir de este momento Elementos del vídeo casero. Construimos una suerte de segundo protocolo de análisis formado por éstos organizados en dos bloques:

Bloque 1: Elementos Esenciales.

Grupo principal de características por su frecuencia entre vídeos caseros; su existencia en un vídeo casero es fundamental.

Bloque 2: Elementos Habituales.

Grupo de características secundarias por no ser tan comunes en los vídeos caseros como las esenciales; su existencia en los vídeos caseros no es obligatoria pero ayuda a diferenciar vídeos cuya identificación pueda generar dudas con los Elementos Esenciales.

A través del examen de estos once elementos, podemos aproximarnos a una identificación del vídeo casero. Ésta consiste en reunir las características que (de entre las once citadas) se registran en el vídeo a designar. Ese grupo de características particulares del vídeo analizado se cotejan con los conjuntos de características básicas del vídeo casero hallados en este trabajo. Estos grupos constituyen conjuntos de características mínimas o básicas que han de existir en un vídeo para poder llamarlo casero. La compatibilidad entre ellas y su combinación se explica en este apartado.

\section{Alternativas Existentes en las Características}

Escogimos el procedimiento de la combinatoria porque las características que se han erigido como más comunes y constantes en el vídeo casero se dan de un modo aleatorio e intermitente.

\subsection{Elementos esenciales}

Los elementos esenciales no tienen por qué darse sin excepción, sino que un vídeo puede ser casero con grupos mínimos de estas características. La compatibilidad entre estos elementos está sujeta a las propias particularidades de cada uno, tal y como se explica a continuación.

- La acción tuvo lugar en la realidad (R),

- La edición de imagen ha sido poco elaborada (IE),

- La edición de sonido ha sido poco elaborada (SE),

- La calidad de imagen es baja (IQ) 
- La calidad de sonido es baja (SQ)

- El vídeo ha sido grabado con webcam (W),

\subsubsection{La acción tuvo lugar en la realidad ${ }^{7}$}

El contenido del vídeo no es fruto de un montaje, manipulación técnica o animación que simule una acción real. Lo acontecido en la obra sucedía en el momento de ser grabada, aunque pudo haberse hecho sólo para que fuera grabado; es decir, ha podido ser teatralizado, pero su ficción no ha sido editada, animada o montada, sino fingida únicamente por sus personajes. El tiempo real de la grabación se corresponde con el tiempo real de la acción que en ella transcurre. Distinguiremos esta característica con el símbolo $R$, inspirado en la inicial de real en inglés, real, que equivale al enunciado:

\section{$R=$ La acción tuvo lugar en la realidad}

\section{Compatibilidades de $R$}

El elemento $R$ es el primero que tomamos como identificativo del video casero. $R$ es compatible con todas las características que conforman la lista de características básicas y habituales, a excepción del elemento habitual $C 2$ (hay cortes durante la grabación y éstos son bruscos) pues se entiende que los cortes durante la grabación alteran la lógica temporal o espacial que se respeta en una acción real. Además, ha de estudiarse detenidamente su relación con los elementos esenciales IE (La edición de imagen es poco elaborada) y SE (La edición de sonido es poco elaborada), que implican un montaje o edición que ha podido modificar la secuencia narrativa natural ${ }^{8}$.

\section{Alternativas a $R$}

Con la exposición de las alternativas a los Elementos Esenciales reflejamos las posibilidades que encontraremos en las fórmulas mínimas del vídeo casero.

Si no se da $R$, ha de darse $I E$ (la pieza registra edición de imagen poco elaborada) o $S E$ (la pieza registra edición de sonido poco elaborada).

Por tanto, es obligatorio que en todo vídeo casero se dé, al menos, R, IE o SE

${ }^{7}$ Ejemplo:

$\mathrm{http} / / / \mathrm{www}$. youtube.com/watch? $\mathrm{v}=$ on3Rtk_AmxQ\&oref=http $\% 3 \mathrm{~A} \% 2 \mathrm{~F} \% 2 \mathrm{Fwww}$.youtube.com\%2Fr esults\%3Fsearch_query\%3Dbirth\%2B11\%26oq $\% 3$ Dbirth\%2B11\%26aq $\% 3 \mathrm{Df} \% 26 \mathrm{aqi} \% 3 \mathrm{D} \% 26 \mathrm{aq1} \% 3 \mathrm{D}$ \%26gs_sm\%3De\%26gs_upl\%3D1331011331010113506111110101010101011010\&has_verified=1

8 Podría darse, asimismo, una convivencia de los parámetros R, IE y SE alternativamente sin que el vídeo deje de ser casero. Tenga en cuenta el analista que la edición de imagen y de sonido puede hacerse sin alterar el tiempo real de la acción. 


\subsubsection{Existe edición de imagen poco elaborada9}

La edición de imagen que se refleja en el vídeo evidencia un trabajo amateur por la escasa o pobre calidad del conjunto de la obra, atendiendo a su montaje, ya sea en cuestiones de ritmo, adecuación de planos o lógica narrativa del montaje, concluyendo que la obra total resulta poco elaborada. Distinguiremos este atributo con el símbolo $I E$, iniciales del inglés image edition, que equivale a:

$I E=$ La pieza registra edición de imagen poco elaborada

Consideraremos éste como uno de los elementos más reveladores de la categoría amateur. De no registrarse $I E$ habiendo montaje o edición, y no darse tampoco el parámetro esencial SE (La edición de sonido es poco elaborada), el vídeo se descarta del género casero.

\section{Compatibilidades de IE}

El elemento $I E$ es compatible con todos los elementos esenciales y habituales del vídeo casero. De la presencia de $I E$ depende la presencia del elemento habitual $C 2$. (Los cortes durante la grabación son bruscos). Este último no puede aparecer si no se da el elemento $I E$.

\section{Alternativas a IE}

Se considera esencial que se dé $I E$ cuando no se dé $R$ (la acción del vídeo tuvo lugar en la realidad) o SE (la pieza registra edición de sonido poco elaborada). Si no se da $R$ ni $S E$, e $I E$ no existe, el vídeo no se considera casero de acuerdo a los criterios que se objetivan en este trabajo.

\subsubsection{Existe edición de sonido poco elaborada ${ }^{10}$}

La edición del sonido es muy básica por haber sido añadida una pista de audio a la imagen o por ensamblarse con un básico programa de montaje. El resultado será poco elaborado aduciendo motivos como la sencillez del conjunto sonoro añadido a la imagen, de manera que la obra opta a la categoría amateur. Distinguiremos este atributo con el símbolo $S E$, del inglés sound edition, que equivale a:

$S E=$ La pieza registra edición de sonido poco elaborada

De haber montaje o edición de sonido y no registrar el vídeo $S E$ ni el elemento $I E$ (La edición de imagen es poco elaborada), la obra sería considerada profesional.

\footnotetext{
${ }^{9}$ Ejemplo: $\mathrm{ex}=24$

http://www.youtube.com/watch?v=N2rZxCrb7iU\&feature=BF\&list=PL6F8670ADD7E584AC\&ind

${ }^{10}$ Ejemplo: http://www.youtube.com/watch?v=cBFvu4FiYds\&feature=fvst
} 


\section{Compatibilidades de SE}

El parámetro $S E$ es compatible con todos los elementos del vídeo casero. En su ausencia, se registrará sin más la baja calidad del sonido con el código del elemento esencial SQ (La calidad del sonido es baja).

\section{Alternativas a $S E$}

$S E$ puede darse o no. Su presencia apoya la pertenencia al género casero al mismo nivel que el elemento esencial IE (la pieza registra edición de imagen poco elaborada) o $R$ (La acción del vídeo tuvo lugar en la realidad), pero la frecuencia de $\mathrm{SE}$ es mucho menor.

\subsubsection{La calidad de la imagen es baja ${ }^{11}$}

La calidad de imagen puede apreciarse en la nitidez visual de la pieza. La calidad de la imagen es baja cuando ésta no se percibe nítida y claramente, sino borrosa, con temblor o torpeza de movimientos. La gradación de la (escasa) nitidez puede variar de unos contenidos a otros, ya que depende del dispositivo con el que ha sido grabada. Distinguiremos esta característica con el símbolo $I Q$, iniciales de image quality, que equivale al enunciado:

\section{$I Q=$ La calidad de imagen es baja}

Se trata de uno de los elementos más importantes para la identificación de vídeos caseros, pero los avances tecnológicos pueden contrariar su importancia. Es posible que vídeos caseros que registren el elemento esencial $W$ ( $E l$ vídeo ha sido grabado con webcam) o el elementos esencial IE (La edición de imagen es poco elaborada) no registren $I Q$. Esto se debe a la precisión de los dispositivos webcam y a los resultados de un vídeo editado, que, aunque simple o poco elaborado, puede tener mejor calidad de imagen que otros.

\section{Compatibilidades de IQ}

La característica $I Q$ es compatible con todas los demás. Puede darse en vídeo casero aunque éste carezca de alguno de estos elementos esenciales: IE (La edición de imagen es poco elaborada), $R$ (La acción tuvo lugar en la realidad), IQ (La calidad de imagen es baja) o $W$ (El vídeo ha sido grabado con webcam). No sería considerada casera una pieza que no registre ninguno de estos elementos.

\footnotetext{
${ }^{11}$ Ejemplo: http://www.youtube.com/watch?v=Ysqh1uzqGrc
} 


\section{Alternativas a $I Q$}

Si $I Q$ no se da, se dará el elemento esencial $W$ (El vídeo ha sido grabado con webcam).

Si no se dan los elementos esenciales $I Q$ ni $W$, habrá que atender a $S Q$ (La calidad del sonido es baja), IE (La pieza registra edición de imagen poco elaborada), SE (la pieza registra edición de sonido poco elaborada) y el elemento habitual $S$ (El director o cámara y el personaje son la misma persona).

\subsubsection{La calidad del sonido es baja ${ }^{12}$}

El sonido llega confuso, con ruido y falta de precisión. Ecos, voces mal definidas, se registran en la obra impidiendo una correcta percepción del audio. Distinguiremos esta característica con el símbolo $S Q$, de sound quality, que equivale al enunciado:

$S Q=$ La calidad del sonido es baja

Como sucede con la calidad de imagen, los elementos sonoros son clave para distinguir a un vídeo casero de otro tipo de vídeos, pero podemos encontrar dispositivos al alcance del aficionado con una alta definición sonora.

\section{Compatibilidades de $S Q$}

El elemento $S Q$ es compatible con el resto. En caso de no registrarse $S Q$, es necesario comprobar SE (La edición del sonido es poco elaborada). Si el sonido de la pieza es de alta calidad, conviene descartar que haya sido editado, y comprobar asimismo esa edición, de manera que desechemos la posibilidad de un trabajo muy elaborado, y así la intervención profesional. De darse el elemento esencial $S E$, habría que atender al resto de elementos. De no darse $S Q$ ni $S E$, el vídeo podría no registrar sonido, por ejemplo. En cualquiera de los casos mencionados es necesario atender al resto de elementos.

\section{Alternativas a $S Q$}

Si no se da $S Q$ ni $S E$, habrá que atender a $I Q$ (La calidad de imagen es baja), W (el vídeo ha sido grabado con webcam), IE (la pieza registra edición de imagen poco elaborada) y a los elementos habituales.

\subsubsection{El video ha sido grabado con webcam ${ }^{13}$}

Todo vídeo grabado con un dispositivo webcam es un vídeo amateur. Para identificar este aspecto hemos de observar atentamente el ángulo de grabación y la escena que tenemos delante, así como la dificultad en la ejecución de los movimientos de cámara.

\footnotetext{
${ }^{12}$ Ejemplo: http://www.youtube.com/watch? $\mathrm{v}=0$ jtxabQfkY0\&feature=related

${ }^{13}$ Ejemplo: http://www.youtube.com/watch? $\mathrm{v}=$ ozJnkWng7os\&feature=related
} 
Las webcams son dispositivos fijos o móviles en el entorno del ordenador o insertos en el monitor del mismo. En numerosas ocasiones encontraremos vídeos profesionales que intenten emular las características de grabación de una webcam, precisamente para poder simular un vídeo casero. Distinguiremos esta característica con el símbolo $W$, inicial de webcam, que equivale a:

$W=$ El video ha sido grabado con webcam

\section{Compatibilidades de $W$}

El elemento esencial $W$ es compatible con todos los demás elementos, esenciales y habituales. Aunque los webcam videos no suelen recoger movimientos de cámara ni cortes, podrían registrarse sin que se descarte la autoría amateur. Es propia de $W$ la ausencia de $I Q$, por la alta definición de las webcams, pero no imposible de combinar.

\section{Alternativas a $W$}

$W$ puede existir sin $I Q$ ( La calidad de imagen es baja), pero si no existe $W$ ni $I Q$, habrá que atender a $S Q$ (La calidad del sonido es baja), IE (La pieza registra edición de imagen poco elaborada), SE (La pieza registra edición de sonido poco elaborada) así como a los elementos habituales $C$ (El corte final es brusco), C2 (La obra registra cortes durante la grabación y éstos son bruscos), $S$ (El director o cámara y el personaje son la misma persona) y PH (No se percibe un trabajo fotográfico previo).

\subsection{Elementos habituales}

No obstante, y dado que los vídeos caseros son creaciones imprevisibles, se ha hecho necesario respetar un grupo de características extra que ayuden a confirmar casos dudosos de vídeo casero, los Elementos Habituales. En este grupo se incluyen los elementos que son habituales en la constitución de vídeos amateur, si bien menos frecuentes que los esenciales y absolutamente prescindibles para que un vídeo sea denominado casero. Su inclusión en la designación desempeña una función auxiliar para aquellas obras que lo precisen. La compatibilidad de estas características entre sí y respecto de las esenciales está reflejada en este apartado.

- La grabación registra movimientos de cámara bruscos $(\mathrm{M})$,

- Los cortes al final de la grabación son bruscos (C),

- Los cortes durante la grabación son bruscos (C2),

- Cámara y personaje son la misma persona (S),

- Existe un trabajo fotográfico previo a la grabación (PH).

Se examinan después de los Elementos Esenciales y la comprobación de su efectividad para la identificación del vídeo casero se hace a través de las combinaciones básicas de características Esenciales y Habituales. 


\subsubsection{Los movimientos de cámara son bruscos ${ }^{14}$}

La brusquedad de los movimientos de cámara no es una característica imprescindible en las piezas caseras, pero se registra en un gran número de ellas. Destacaremos la torpeza en la ejecución del dispositivo como propia de la obra amateur. Distinguiremos este elemento con el símbolo $M$, de movement, que equivale al enunciado:

$M=$ La obra registra movimientos de cámara y éstos son bruscos

Conviene señalar que existe una multitud de vídeos domésticos que muestran gran delicadeza en el manejo de la cámara.

\section{Compatibilidades de $M$}

El elemento $M$ tiene combinatoria con todos los elementos del vídeo casero, incluido $W$ (El vídeo ha sido grabado con webcam), cuya relación (como apuntábamos anteriormente) puede despertar dudas por la ubicación (inserta en la pantalla del ordenador) de muchos dispositivos webcam.

\section{Alternativas a $M$}

$M$ puede darse o no. Su presencia, apoyada por los elementos esenciales, contribuye a diferenciar si el vídeo es casero al mismo nivel que el resto de elementos habituales, aunque su presencia en los vídeos caseros es bastante común.

\subsubsection{El corte final es brusco ${ }^{15}$}

El corte final se da en toda pieza videográfica y es propio de vídeo casero que sea brusco, inesperado. No obstante, puede darse un corte cuidadoso en un vídeo amateur. Distinguiremos esta característica con el símbolo $C$, del inglés cut, que equivale al enunciado:

$C=$ El corte final es brusco

\section{Compatibilidades de $C$}

El elemento $C$ puede registrarse o no en todos los vídeos caseros, independientemente de otros elementos existentes en esa pieza.

\section{Alternativas a $C$}

De registrarse, confirma la pertenencia del vídeo al género casero al mismo nivel que los otros elementos habituales. Es el más común de este bloque.

\footnotetext{
14 Ejemplo: http://www.youtube.com/watch?v=521prmXNIA4

15 Ejemplo: http:/www.youtube.com/watch?v=txqiwrbYGrs\&feature=rec-LGOUT-exp_fresh+div1r-2-HM
} 


\subsubsection{Los cortes durante la grabación son bruscos ${ }^{16}$}

Del mismo modo, puede registrarse brusquedad en el corte entre toma y toma de una pieza con montaje de imagen o de sonido. El salto o ensamblaje de imágenes es notable brusco y esperado en los vídeos caseros que registren edición. No obstante, pueden darse vídeos caseros cuyos cortes durante la grabación se hayan ejecutado con cuidado, sin torpeza o brusquedad en los cambios de plano o escena ${ }^{17}$.

Distinguiremos esa característica con el símbolo $C 2$, de cut con el número 2 para que no sea confundido con $C$ (El corte final es brusco), y equivale al enunciado:

\section{$C 2=$ La obra registra cortes durante la grabación y éstos son bruscos}

Atenderemos al resultado final de la edición de la pieza, desde el punto de vista de la imagen y del sonido (IE, $S E)$.

\section{Compatibilidades de $C 2$}

El elemento $C 2$ es registrable en vídeos que, a su vez, incluyan los elementos esenciales $I E \mathrm{y} /$ o $S E$. No obstante, los vídeos que registren $I E$ y/o $S E$ no tendrán por qué incluir $C 2$ entre sus características. $C 2$ no se dará con el elemento esencial $R$, son incompatibles.

\section{Alternativas a $C 2$}

El elemento habitual $C 2$ puede darse o no. De existir, se registrará también $I E$ ( $L a$ edición de imagen es poco elaborada). Si se da $C 2$ pero no existe $I E$, el vídeo no es casero, según los criterios que se objetivan en este estudio

\subsubsection{Director (cámara) y personaje son la misma persona ${ }^{18}$}

El director o cámara y el personaje coinciden en una misma persona. Distinguiremos este elemento con el símbolo $S$, del inglés same (mismo) que equivale al enunciado:

$S=$ El director o cámara y el personaje son la misma persona

\footnotetext{
16 Ejemplo:

http://www.youtube.com/watch?v=N2rZxCrb7iU\&feature=BF\&list=PL6F8670ADD7E584AC\&ind $\mathrm{ex}=24$

${ }^{17}$ Se denomina así al salto que la imagen realiza cuando se efectúa un corte con motivo de un cambio en la toma del plano (se graba otra cosa en la misma escena), o por el fin de una escena concreta (la escena anterior termina y se corta para filmar la siguiente). Los cambios de plano, escena y secuencia son notables en el visionado final de un texto fílmico o videográfico y ayudan a la construcción de la historia y a su mejor comprensión. En vídeo casero, estos cambios suelen ser muy básicos y corresponden a verdaderos saltos que realiza la cámara para cambiar de imagen.

${ }^{18}$ Ejemplo: http://www.youtube.com/watch?v=xz1 fRYlf9Nk
} 
No suele darse en vídeo profesional, pero podemos encontrarlo en cine dogma, reality shows y otros formatos en TV.

\section{Compatibilidades de $S$}

El elemento $S$ es combinable con todos los parámetros de vídeo casero. Podría darse en vídeo profesional y podría no darse en vídeo casero; es imprescindible atender al resto de características. Este elemento coincidirá normalmente con $W$, por las características del dispositivo webcam.

\section{Alternativas a $S$}

$S$ puede darse o no. De registrarse, justifica la pertenencia del vídeo al género casero y contribuye a su identificación como tal. Se da $S$ en la mayor parte de los homedances $^{19}$ y los videoblogs $s^{20}$, por lo que su presencia es destacable en el vídeo casero que se produce actualmente.

\subsubsection{No existe un trabajo fotográfico previo ${ }^{21}$}

No puede registrarse un trabajo fotográfico profesional previo a la grabación que valore la utilización de la luz en favor de una mejor estética del resultado en un vídeo de autoría amateur. Distinguiremos esta característica con el símbolo $\mathrm{PH}$, de photo que equivale al enunciado:

\section{$P H=$ No se percibe un trabajo fotográfico previo}

Algunos vídeos caseros pueden registrar un cuidado básico con la luz que no es propiamente un trabajo fotográfico, pero puede igualarse al obtenido en algunos vídeos profesionales. Se trata, por tanto, de una característica difícil de identificar. Es necesario, en el caso de que despierte dudas, atender al resto de elementos habituales.

\section{Compatibilidades de $\mathrm{PH}$}

El elemento $P H$ es combinable con todos los demás de la lista.

\section{Alternativas a $P H$}

PH puede darse o no. Su presencia contribuye a que el vídeo sea casero. Es una característica común en todos los vídeos caseros, aunque su identificación no siempre resulta fácil. Es necesario atender primero al resto de elementos habituales, razón por la cual es el último de este grupo.

${ }^{19}$ Ejemplo: http://www.youtube.com/watch?v=Ysqh1uzqGrc

${ }^{20}$ No así las filmaciones que el aficionado haya podido realizar de una emisión televisiva, por internet o cinematográfica de dichos contenidos.

${ }^{21}$ Como su nombre indica, un vídeo corporativo está realizado para difundir, publicitar o explicar el trabajo de una compañía, empresa o corporación para que sus socios, miembros y clientes lo conozcan. 
Ninguna característica registrada en el primer o el segundo grupo es exclusiva de vídeo profesional, sino que ha de interpretarse combinada con otras, constituyendo conjuntos de características básicas de vídeo casero. Hemos denominado a estas combinaciones

\section{DEFINICIÓN DEL VÍDEO CASERO}

\subsection{La identificación}

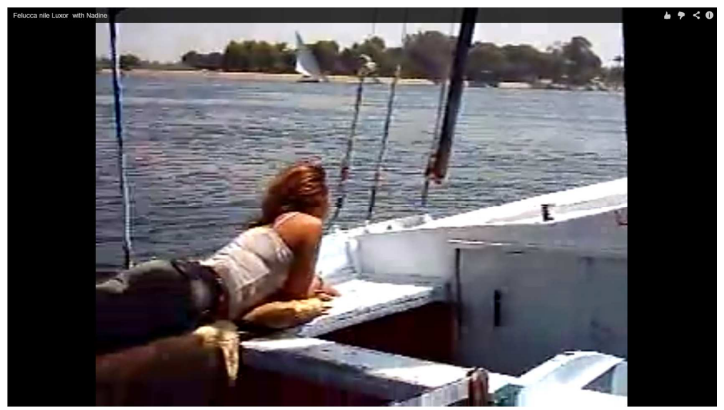

Imagen 4 :Felucca Nile Luxor with Nadine

Para llevar a cabo la identificación del vídeo casero, proponemos el siguiente procedimiento:

- Se observarán, en primer lugar, qué elementos esenciales, de los seis propuestos, se registran en la pieza, observando si la definición de cada elemento se da en el vídeo en cuestión.

- Se llevará a cabo la misma comprobación con los elementos habituales.

- Con los caracteres obtenidos, elaboraremos la fórmula equivalente a la pieza. Por ejemplo, si en el vídeo se registran los elementos R, IQ, SQ y PH, la fórmula correspondiente a ese vídeo sería $\mathrm{R}+\mathrm{IQ}+\mathrm{SQ}+\mathrm{PH}$.

- Comprobaremos, gracias a las compatibilidades expuestas para cada elemento, si el vídeo es casero.

Dicho procedimiento ha sido escogido por combinar el registro de unas características que pueden aparecer en piezas de cualquier género audiovisual con otras específicas del género casero. El sometimiento a todos los elementos del vídeo casero contempla una revisión global del contenido, evitando abarcar casos de vídeos profesionales aparentemente caseros, como sucedería en una definición cerrada. No hay que olvidar que los elementos reflejados en estos resultados no pueden aplicarse a cualquier género audiovisual, sino sólo a aquellos en relación a cuyo género se tengan 
dudas. Con esta aclaración se intentan excluir productos reconocidamente profesionales, cuya autoría puede conocerse a la perfección. Los elementos del vídeo casero se han escogido para ser aplicados a aquellas piezas que "parecen caseras" para saber si lo son o no. Quedan excluidos todos los vídeos, piezas, obras o grabaciones videográficas cuyo contenido audiovisual sea profesional, producido en el medio televisivo, cinematográfico o Internet, englobando los siguientes:

\section{Publicidad}

- Vídeos corporativos o fragmentos de vídeos corporativos,

- Campañas audiovisuales de propaganda y marketing ${ }^{22}$ de entidades, organizaciones, asociaciones, etc... o fragmentos de campañas audiovisuales de propaganda y marketing de entidades, organizaciones, asociaciones, etc...,

- Spots $^{23}$ publicitarios o fragmentos de spots publicitarios,

- Emplazamientos publicitarios de marca o fragmentos de emplazamientos publicitarios de marca.

Televisión (se entienden los siguientes productos aparecidos en este medio):

- Programas o fragmentos de programas,

- Series o fragmentos de series,

- Largometrajes, documentales, cortometrajes o fragmentos de largometrajes, documentales, cortometrajes ${ }^{24}$,

- Anuncios publicitarios o fragmentos de anuncios publicitarios,

- Concursos o fragmentos de concursos,

- Shows o fragmentos de shows,

- Informativos o fragmentos de informativos,

- Videoclips o fragmentos de videoclips,

- Otros formatos televisivos o fragmentos de otros formatos televisivos.

Cine (se entienden los siguientes productos aparecidos en soporte cinematográfico, ya sea éste digital o analógico):

- Largometrajes o fragmentos de largometrajes,

\footnotetext{
${ }^{22}$ Se entiende por campaña de marketing el despliegue de medios y estrategias publicitarias que una empresa, marca o compañía lleva a cabo para promocionar sus productos.

${ }^{23}$ Cortometraje comercial de 20 a 60 segundos destinado a captar la atención del espectador.

${ }^{24} \mathrm{El}$ caso de las grabaciones pirata que circulan por la red y que consisten en una grabación (en ocasiones íntegra, de la duración exacta del original) de un film que se emite en una sala de cine, constituye según esta investigación y quienes la hemos realizado, una pieza casera, pues ha sido realizada con un dispositivo doméstico. Que sus fines estén destinados a mover su producto con la misma función que el producto profesional al que sustituye por diversas razones (inmediatez, rapidez y economía), no lo convierte en un producto audiovisual profesional. Sí lo es, indiscutiblemente, la pieza que se graba en él, pero no esa copia.
} 
- Cortometrajes o fragmentos de cortometrajes,

- Documentales o fragmentos de documentales,

- Otro tipo de muestra cinematográfica o fragmentos de la misma.

\section{Sobre el Videoarte}

Categoría artística surgida como consecuencia de la consolidación de los medios de masas, se diferencia del cine experimental en que no cumple con las convenciones del cine, carece de narrativa y guión y está realizado en vídeo, por lo que es una alternativa económica. Se trata de una narración conceptual que sintetiza códigos expresivos de distintos ámbitos del audiovisual, rompiendo con los parámetros convencionales y sirviéndose de unos nuevos. Su producción, imprevisible e inclasificable, puede confundirse con piezas de vídeo casero (incluso puede estar hecha por una o varias piezas de vídeo casero). Los elementos hallados excluyen un género como éste, pero la ausencia de límites en la realización del videoarte lo convierte en una pieza que, a menudo, puede parecer un vídeo casero. Incluso podría superar una análisis de los elementos y figurar entre los conjuntos de características básicas. Es importante tenerlo en cuenta como excepción.

\section{CONCLUSIÓN}

\subsection{Utilidad de la investigación}

Como ha ocurrido en otras muchas ocasiones, nos encontramos ante un fenómeno novedoso, que ya ha tenido una cierta evolución antes de ser abordado científicamente. Estudiar el vídeo casero suponía introducirse en un terreno virgen, inexplorado pero continuamente utilizado, citado y ya, maduro. No tenemos la pretensión de encerrar el contenido en unas normas. El vídeo casero abarca su propia historia y ha de atender al único factor común que perdura en el tiempo: su autor. En base a eso, consideramos suficientes las fórmulas halladas, pues contemplan esta característica por encima de todas las demás. Es este mismo aspecto el que nos ha hecho volver a la consideración previa que sentaba lo conocido sobre vídeo casero: estas piezas siguen siendo obras de baja calidad, huella de la falta de profesionalidad de sus autores. Así, si al pensar en el vídeo casero en los 90 podíamos tender a pensar en escenas determinadas que caracterizaban el género, hoy no podemos fijar normas de contenido. Es por eso que todos los criterios que hemos considerado válidos atañen exclusivamente a la grabación, su ejecución y las características de su resultado.Lo fascinante de una revolución como la del vídeo casero mira hacia esta emergencia de obras y su poder. Es destacable que la tecnología aplicada a los dispositivos de grabación y a la práctica de la difusión genere en el usuario (hasta entonces pasivo) una capacidad extraordinaria por expresarse de forma audiovisual y contribuir a un movimiento sociocomunicativo que no cesa. Asimismo, es de señalar que esos productos cobren un crédito e invadan los medios tradicionales, pero, ¿a qué se debe? ¿Se ha perdido todo criterio sobre los contenidos audiovisuales? El vídeo casero 
actual es producido por el portador de un dispositivo de grabación en el bolsillo, disponible en cualquier momento y cualquier lugar. Esto hace que todo usuario se convierta en un operador de cámara potencial que puede grabar cualquier escena que suceda, de interés público o privado. Hoy, muchos medios de comunicación hacen primar entre sus contenidos esa inmediatez, sorpresa o espontaneidad que caracteriza los vídeos amateur y es gancho de audiencia (televisiva o de Internet). La calidad queda a un lado y, a veces, el criterio de selección también.

Esta apertura de los medios profesionales a los contenidos caseros a menudo recuerda a una carrera por el éxito más que por la noticia más fresca y real. El vídeo casero no nace con reglas y no está sujeto a una publicación con reglas, pero su difusión indiscriminada puede confundir al espectador (que a la vez lo produce) si la gestión de los medios profesionales no es responsable. La lectura del realismo en los vídeos caseros es explotada por las agencias de comunicación y publicidad por el beneficio que esta tendencia puede ofrecerle, pero el estilo se agota y la copia cansa. El espectador manifiesta sus preferencias, y éstas miran (también) hacia el contenido amateur. Sin embargo no es ésta suficiente razón para exprimir el género y desvirtuar sus orígenes. El fenómeno de la viralidad, objeto de la presente investigación de la autora de este artículo, contempla cómo el consumo de piezas en Internet responde a claves ocultas que dependen en gran parte de este usuario, y que las productoras comerciales tratan de manejar para confundir al espectador y extraer con ello un reporte. Tal vez fuera positivo que los medios escucharan, observaran, vieran qué es lo que el espectador está demandando con los contenidos que ahora se atreve a realizar, con aquellos que consume, y cuál es, asimismo, el lugar en que le ha colocado una asimilación de décadas de la imagen audiovisual y los contenidos profesionales, y que extraiga de estos hechos un precedente de creatividad, de poder y de nueva creación profesional, de evolución en la educación del espectador, y no se limiten a copiar fórmulas que funcionen.

El espectador (hoy nuevo creador) evoluciona, y todo lo que demande y ofrezca irá en función de esa evolución, en la que tienen un papel fundamental los medios de comunicación profesionales. Puede resultar arriesgado aventurar que el vídeo casero no se agotará en lo previsible ni dejará de ser producido. El usuario hoy quiere conservar y compartir su realidad y ha encontrado una forma sencilla y satisfactoria de hacerlo. Esta seguridad está basada en lo aprendido y observado en el proceso de este trabajo. Es precisamente la necesidad por comunicar, por difundir y la creada con las redes sociales la que nos despierta un afán por descubrir qué hay detrás de este cambio y esta expansión de contenidos. 


\section{BIBLIOGRAFÍA}

ARONOWITZ, S., MARTINSONS, B. y MESER, M. (1998): Tecnociencia y cibercultura. Barcelona: Paidós.

BARTHES, R. (1970): Introducción al análisis estructural de los relatos. Buenos Aires: Editorial Tiempo Contemporáneo.

CEAC S.A. Colección CEAC Imagen.

COLLAR, A. (2009): La nueva videocreación. Madrid: Actas del I Congreso internacional Sociedad Digital.

CRIADO, M.(2004): "Historia de la videocámara" Suplemento ARIADNA para El Mundo Digital. 20 de Junio de 2004. Número192. Disponible en: http://www.elmundo.es/ariadna/2004/192/1087993918.html

GONZÁLEZ REQUENA, J.(2006): Clásico, manierista, postclásico. Los modos del relato en el cine de Hollywood. Valladolid: Castilla Ediciones, Colección Trama yFondo.

JENKINS, H. (2008): Convergence of Culture. Barcelona: Paidós.

MACRAE, K. (2006) Digital contra analógico: El juego ha terminado. Barcelona:

MANOVICH, L. (2005): El lenguaje de los nuevos medios de comunicación. Barcelona: Paidós.

MARTÍNEZ, J., VILA I FUMÁS, P. y otros. (2004): Manual básico de tecnología audiovisual y técnicas de creación, emisión y difusión de contenidos. Barcelona: Paidós.

PINDADO, J. (2006): Los medios de comunicación y la construcción de la identidad adolescente. Bilbao: Zer, Revista de Estudios de Comunicación, Universidad del País Vasco. Disponible en: http://www.ehu.es/ojs/index.php/Zer/article/view/3712

PIÑUEL,J. (2002): Epistemología,metodología y técnicas del análisis de contenido. Estudios de Sociolingüística 3 (1), pp 1-42. Consultado el 27 de Septiembre de 2010. Disponible en: $\mathrm{http} / / / \mathrm{www} . \mathrm{ucm} . \mathrm{es} / \mathrm{info} / \mathrm{mdcs} / \mathrm{A}$.Contenido

PIÑUEL, J. (1978): Un análisis de contenido de devociones populares. Revista Española de Investigaciones Sociológicas. 3/78, pp 135-163. Consultado el 10 de Septiembre de 2010.

PROPP, V. (1928) Morfología del cuento. Madrid: Editorial Fundamentos.

Disponible en: dialnet.unirioja.es/servlet/fichero articulo? codigo $=665837$

SAN CORNELIO, G. y ARDÉVOL, E. (2007): Si quieres vernos en acción: YouTube.com! Santiago de Chile: Revista chilena de Antropología visual, $\mathrm{n}^{\circ}$ 10,Páginas 1-29. Consultado el 15 de Abril 2010. Disponible en: http://www.antropologiavisual.cl/imagenes10/imprimir/ardevol_\&_san_cornelio.pdf SÁNCHEZ CARRIÓN, J. (1978): Análisis estructural del Telefilm. Revista Española de Investigaciones Sociológicas 3/78 pp. 95-110. Consultado el 15 de Septiembre de 2010. Disponible en: dialnet.unirioja.es/servlet/fichero_articulo?codigo $=665833$

VV.AA. (2009): The YouTube reader, Snickars y Vonderau. Suecia: Mediehistoriskt. 
WHITE, G. (1988): Técnicas del vídeo $2^{a}$ Edición. Madrid: Instituto Oficial de Radio y Televisión, Heinemann Newnes.

\section{REFERENCIAS VIDEOGRÁFICAS}

Bebe Bob Marley By MaIkYxXx100 HD [contenido generado por el usuario, on-line] Cread. Mayki Rast 10/11/2010. 1 min 14 segs

http://www.youtube.com/watch?v=521 prmXNIA4 (Consultado el $17 / 10 / 2011)$

Birth 11 [contenido generado por el usuario, on-line]. Cread. future midwife. 21/01/2008. 4 mins. 21 segs.

http://www.youtube.com/watch?v=on3Rtk_AmxQ\&oref=http\%3A\%2F\%2Fwww .youtube.com $\% 2$ Fresults $\% 3$ Fsearch_type $\% 3$ Dvideos $\% 26$ search_query $\% 3 \mathrm{DHOM}$ E\%2BVIDEO\%26search_sort $\% 3$ Dvideo_view_count $\% 26$ suggested_categories $\% 3$ $\mathrm{D} 23 \% 252 \mathrm{C} 10 \% 252 \mathrm{C} 26 \% 26 \mathrm{page} \% 3 \mathrm{D} 7 \&$ has_verified $=1 \&$ oref $=\mathrm{http} \% 3 \mathrm{~A} \% 2 \mathrm{~F} \% 2 \mathrm{~F}$ www.youtube.com\%2Fverify_age \%3Fnext_url $\% 3 \mathrm{Dhttp} \% 253 \mathrm{~A} \% 2 \mathrm{~F} \% 2 \mathrm{Fwww}$.yo utube.com\%2Fwatch\%253Fv\%253Don3Rtk_AmxQ (Consultado el 11/09/2011)

CitröenDS4 [spotpublicitario] Citröen. 9/06/2011 http://www.youtube.com/watch?v=O32JfS9v3sE (Consultado el 17/09/2011)

Chinese Backstreet Boys - That Way [contenido generado por el usuario, on-line]. Cread. ewo 04/11/2005. 4 mins 56 segs http://www.youtube.com/watch?v=N2rZxCrb7iU\&feature=BF\&list=PL6F8670A DD7E584AC\&index $=24$ (Consultado el 18/09/2011)

Chubby cuppy cake Boy [contenido generado por el usuario, on-line]. Cread. leogunawan 17/10/2008. 45 segs http://www.youtube.com/watch?v=Ysqh1uzqGrc(Consultado el 10/09/2011)

Contigo no, bicho. [Contenido generado por el usuario, on-line]. Cread. Aynmusic. 14/04/2008 3min, 28 segs. http://www.youtube.com/watch?v=OmgYYTZzxbw (Consultado el 8/09/2011).

David After Dentist. [Contenido generado por el usuario, on-line]. Cread. booba1234.30/01/2009. 2 mins.

http://www.youtube.com/watch?v=txqiwrbYGrs\&feature=rec-LGOUTexp_fresh+div-1r-2-HM (Consultado el 17/09/2011).

Felucca nile Luxor with Nadine [contenido generado por el usuario, on-line]. Cread. daddycooldriver. 15/03/2007. 16 segs.

http://www.youtube.com/watch?v=0jtxabQfkY0\&feature=related. (Consultado el $15 / 09 / 2011)$

Hey Soul Sister(Me Singing) [contenido generado por el usuario, on-line]. Cread. keenan cahill 29/05/2010. 3 mins. 31segs

http://www.youtube.com/watch?v=ozJnkWng7os\&feature=related

(Consultado el 5/09/2011) 
Husky Dog Talking - " I love you "[contenido generado por el usuario, on-line]. Cread. gardea23. 07/02/2008 48 segs http://www.youtube.com/watch? $\mathrm{v}=\mathrm{qXo3NFqkaRM}$ (Consultado el 12/09/2011)

La caida del Hombre Arana version corta [contenido generado por el usuario, online]. Cread. erik calvo salazar. 02/09/2007. 27segs.

http://www.youtube.com/watch? $\mathrm{v}=\mathrm{cBFvu} 4 \mathrm{FiYds} \&$ feature=fvst (Consultado el 09/10/2011)

La Duquesa luce bikini de vacaciones en Ibiza [contenido generado por un canal de contenidos]. Cread. canalgente 03/11/2010. 1 min. 25segs.

http://www.youtube.com/watch?v=dSRt0JSBN_M

(Consultado el 10/10/2011)

Matias el humilde [contenido generado por el usuario, on-line]. Cread. pacorro gaeta. 19/11/2006. 1 min. 45segs. http://www.youtube.com/watch?v=IFqEZRzFK9Y

(Consultado el 09/10/2011)

Miedo [contenido generado por el usuario, on-line]. Cread. jason maarquez. 27/03/2007. 53segs. http://www.youtube.com/watch?v=tg2W4tLhOko

(Consultado el 05/09/2011)

My cat tiggy talking saying hello Cread. tiggy talkingcat. 22/07/2007. 27segs. http://www.youtube.com/watch?v=eV71 mpbvl-g (Consultado 05/09/2011)

My humps [contenido generado por el usuario, on-line]. Cread. love handle of leaks. 30/09/2009. 2 mins. 35segs

http://www.youtube.com/watch?v=5gGDIxeKgdU (Consultado el 10/09/2011)

Niña enamorada de su profesor [contenido generado por el usuario, on-line]. Cread krankisimo 12/01/2012. 1 mins. 03segs

$\mathrm{http} / / / \mathrm{www} \cdot$ youtube.com/watch?v=vW1i_d8xxM\&feature=fvst (Consultado el 08/03/2012)

Niña se altera por no cantar con el tono de voz correcto [contenido generado por el usuario, on-line]. Cread. lady mellbourne 13/04/2011. 1 mins. 34segs.

http://www.youtube.com/watch?v=yX2ebw3D7m0 (Consultado el 12/09/2011)

Rave brutal en el campo

Single Ladies Baby, Beyonce Kanye was right CNN [contenido generado por el usuario, on-line]. Cread.zeroprice. 26/09/2009. 51segs

http://www.youtube.com/watch? $\mathrm{v}=\mathrm{xSdZ6akE02I \& feature=related} \mathrm{(Consultado} \mathrm{el}$ $10 / 09 / 2011)$

Stalking cat [contenido generado por el usuario, on-line]. Cread. lowdope. 07/09/2008. 1 min. 14segs.

http://www.youtube.com/watch?v=fzzjgBAaWZw. (Consultado el 10/09/2011)

Tokyo earthquake as it happened 3/11/11 [contenido generado por el usuario, on-line] Atomicboyx 10/03/2011. 2 min 46 segs

http://www.youtube.com/watch?v=xz1fRYlf9Nk (Consultado el 19/09/2011). 